\title{
The Impact on Dimensional Accuracy of Spinning Products Given by Top End Force
}

\author{
Yu YANG \\ Changchun University of Science and Technology, Changchun, Jilin province, China \\ yangyu1983@sina.com
}

Keywords: Trail Top Force, Deformation, Parameter Amended, Size Accuracy.

\begin{abstract}
In the spinning process of aluminum spinning products, the trail top force must be moderate. If trail top force is too small, sulg will be relative rotation with core model, even cast from working area and if trail top force is too strong, it will cause bearing overload of principal axis and trail top. Moderate tail top force can also cause a certain amount of deformation to aluminum products. This deformation will change reduction amount during the spinning phase and make the calculation of spinning pressure that force on rotary wheel not accurate, thus affect the whole spinning craft parameter setting, reducing the size accuracy of the spinning products. This article will carry on the simulation analysis through the deformation of slug on the trail top force and determine the relationship between the trail top force and the amount of reduction during the spinning stage. On the basis of large of craft experiments and simulation, the parameters of the Thamasett algorithm is be amended and this makes the new algorithm that calculate the spinning pressure that force on rotary wheel more accurate. Using the results of new calculation algorithm spinning pressure to set spinning craft parameter, we carry on craft experiment to aluminum blank. And the results show that taking consideration of variation of the reduction amount caused by the trail top force, the size accuracy of aluminum spinning products is slightly increased.
\end{abstract}

\section{Introduction}

Spinning processing refers to the blank installated clip on core model spinning following it, at the same time, making use of spinning tool rolls blank rotating, relative feeding between spinning tool and core model. This makes blank pressed and produce a continuous local plastic deformation, to get back the plastic process of hollow rotation part ${ }^{[1-2]}$. It has been widely used at national defense industry and civil industry aspects. Along with the requirements of size accuracy of spinning products more and more high, the trail top force to blank, especially aluminum blank the effect of extrusion deformation attracting more and more attention.

In the spinning process, it needs to trail top force withstand blank to mandrel. Its upsetting force must be enough, or in the spinning process blank will happen to relative rotation under spinning pressure and centrifugal force, and may even blank cast from working area leading to accidents. Too strong upsetting force not only can cause bearing overload of principal axis trail top, but also cause a certain deformation of aluminum blank ${ }^{[3-4]}$. The change of the deformation will directly influence the accurate setting of spinning craft parameters. Thus affect the dimension precision of entire spinning products, especially the dimension precision of spinning phase. At present, the spinning enterprise at home and abroad all ignore the deformation that caused by trail top force to aluminum blank. This article optimizes spinning pressure and other craft parameters through the analysis of deformation caused by the minimum trail top force, and finally confirm the dimension precision influence of spinning products caused by the trail top force.

This article has a simulation analysis to the deformation of aluminum blank caused by trail top force and calculates and simulates variation of spinning pressure that forced on rotating wheel caused by deformation, the spinning craft experiment shows that taking consideration of deformation of the spinning products caused by the trail top force, the dimension accuracy of spinning products is higher than that without consideration. 


\section{Spinning Equipment Structure and Working Principle}

Spinning machine mainly consists of spindle box, main shaft, core mold, main lathe bed, side lathe bed, cross platform, rotary slide wheel, tail top, heating system, cooling system, hydraulic system and the electrical system and other many parts, as shown in figure 1 below. Spinning machine mainly consists of spindle box, main shaft, core mold, main lathe bed, side lathe bed, cross platform, rotary slide wheel, tail top, heating system, cooling system, hydraulic system and the electrical system and other many parts.

Working principle of cylindrical spinning processing: the blank installated clip on core model is spinning following it, at the same time, making use of rotary wheel rolls blank rotating, relative feeding between spinning tool and core model. This makes blank pressed and produce a continuous local plastic deformation, to get back hollow rotation part ${ }^{[5-6]}$.

\section{Calculation of the Tail Jacking Force and Influence of Reduction Thickness Caused by Tail Jacking Force}

The calculation of tail jacking force is aimed at the blank relatively dormant to core mode in the spinning process. The computation formula that jacking force of tail top is:

$$
Q \geq \frac{3\left[P_{l}\left(R_{0}+e\right)+M_{\sigma}\right]}{2 \mu R_{0}}-P_{Z}
$$

Among them: $P_{l}$ tangential component of spinning pressure; $R_{0}$ induction radius; $e$ scale factor; $\mu$ friction coefficient; $M_{\sigma}$ friction torque of friction force to the rotary center; $P_{Z}$ the axial component of spinning pressure. Experimental parameters are such as shown in table 1.

We can get the following results by establishing finite element model analysis: under top jacking force, the deformation of aluminum blank in radial direction is $0.047 \mathrm{~mm}$, as shown in figure 1 . So the deformation numerical value in spinning initial stages should be $10.647 \mathrm{~mm}$, rather than $10.6 \mathrm{~mm}$.

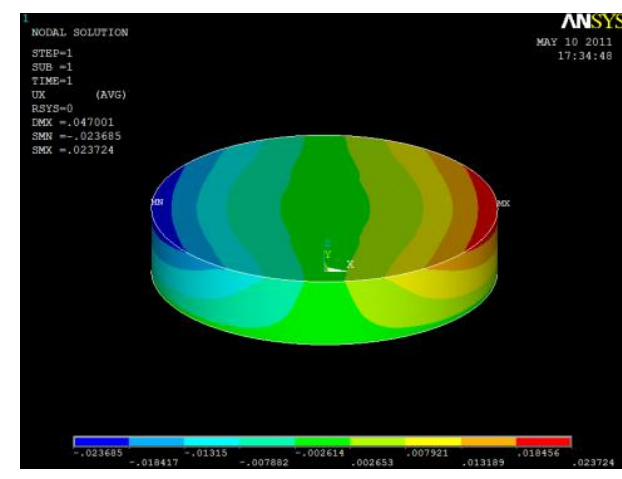

Fig.1 Deflection of Blank Radial Direction

Tab.1 Experimental Parameters

\begin{tabular}{|c|c|c|}
\hline \multirow{2}{*}{ blank } & Parameters & Numerical value \\
\cline { 2 - 3 } & external diameter & $100 \mathrm{~mm}$ \\
\cline { 2 - 3 } & thickness & $20 \mathrm{~mm}$ \\
\cline { 2 - 3 } & length & $100 \mathrm{~mm}$ \\
\hline \multirow{3}{*}{ rotation wheel } & materials & 6061 \\
\cline { 2 - 3 } & diameter & $200 \mathrm{~mm}$ \\
\cline { 2 - 3 } & fillet radius & $10 \mathrm{~mm}$ \\
\hline \multirow{2}{*}{ core mode } & rotation wheel installation angle & $30^{\circ}$ \\
\cline { 2 - 3 } & diameter & $60 \mathrm{~mm}$ \\
\hline \multirow{3}{*}{ Feedrate } & length & $280 \mathrm{~mm}$ \\
\cline { 2 - 3 } & spindle speed & $50 \mathrm{r} / \mathrm{min}$ \\
\cline { 2 - 3 } & feedrate speed & $3 \mathrm{~mm} / \mathrm{r}$ \\
\cline { 2 - 3 } & reduction thickness & $10.6 \%$ \\
\hline
\end{tabular}




\section{Influence of Reduction Thickness to Spinning Pressure}

On the basis of the revised algorithm, Model spinning simulation experiment. The equipment parameters and experimental parameters are as the same as table 1. First, making use of the CATIA to establish geometric model, Deal with geometric model with hypermesh before, as shown in figure 2 .

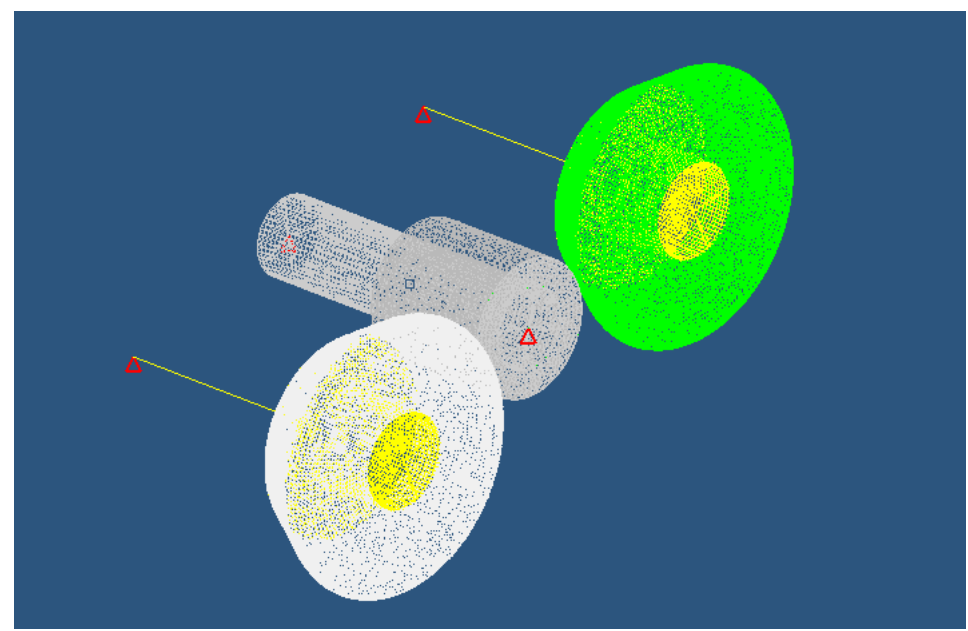

Fig.2 Hypermesh Dealing with Before

Using ansys algorithm to solve, get Spin the wheel for radical force. As shown in figure 3.Axial pressure spin is as shown in figure 4 Spin pressure to cut is as shown in figure 5.

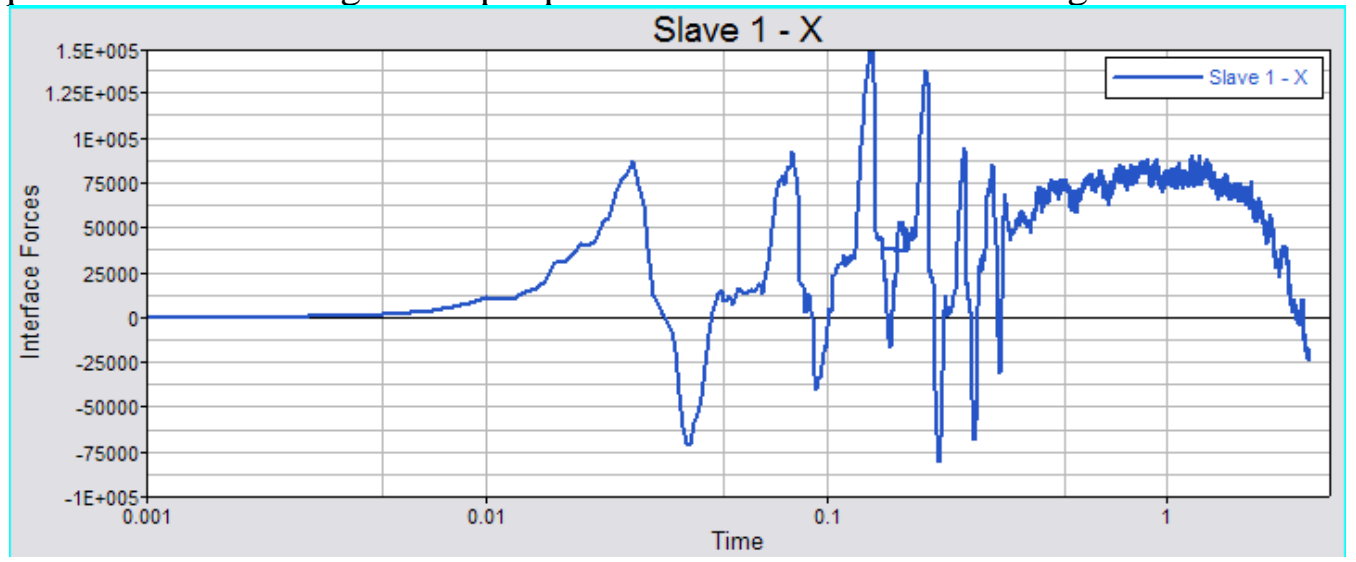

Fig.3 Radial Spinning Pressure

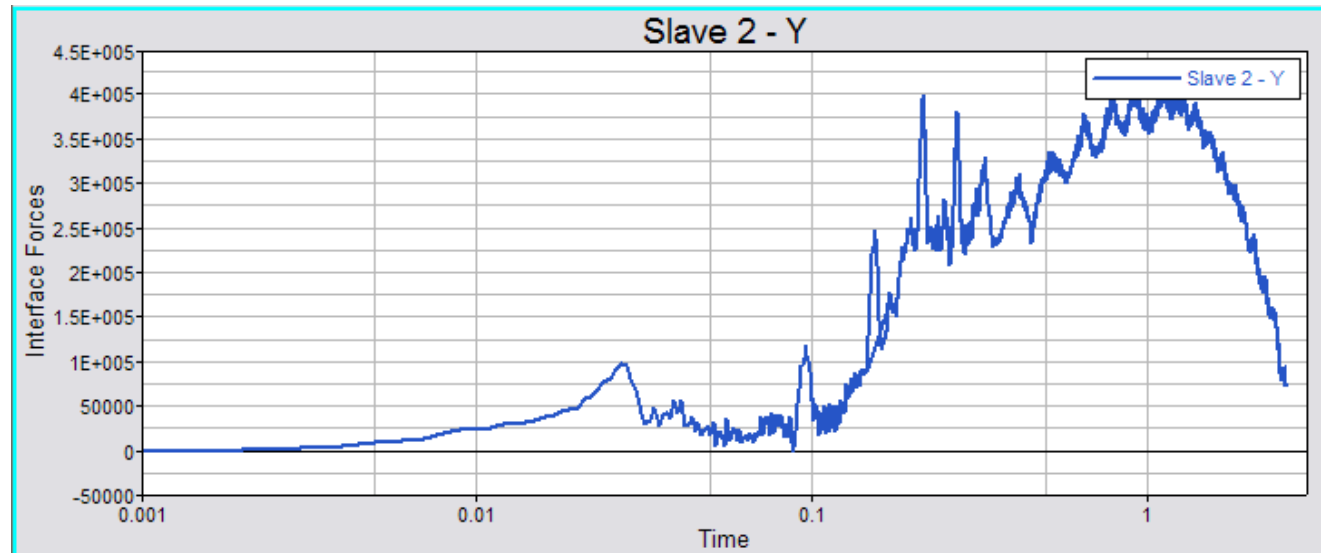

Fig.4 Axisal Spinning Pressure 


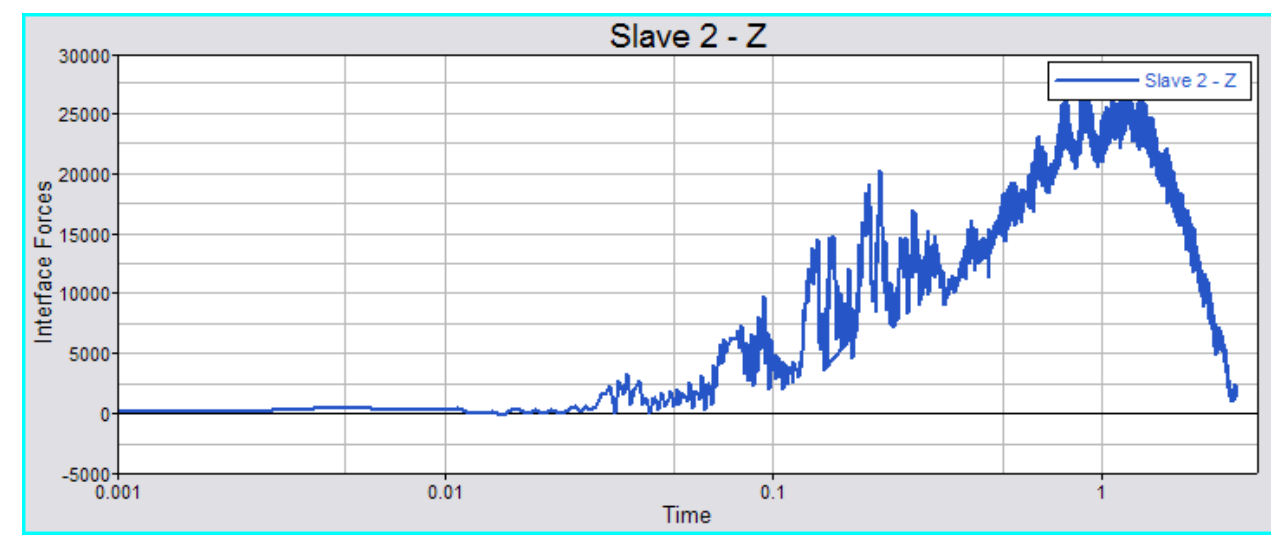

Fig.5 Tangential Spinning Pressure

\section{Spinning Craft Experiment}

We respectively have the spinning craft experiments on considering influenced by tail jacking force and without considering influenced by tail jacking force through making use of the same spinning machine for the same type aluminum blank. And according to the spinning pressure forced on the rotary wheel that calculated by the new algorithm to reset spinning craft parameters. The experimental results are as shown in table 2 .

Tab.2 Experimental Results

\begin{tabular}{|c|c|c|}
\hline & Diameter size precision & Surface roughness \\
\hline \multirow{3}{*}{$\begin{array}{c}\text { Considering spinning craft } \\
\text { experiment influenced by tail }\end{array}$} & $0.301 \mathrm{~mm}$ & 6.3 \\
\cline { 2 - 3 } jacking force & $0.300 \mathrm{~mm}$ & 6.3 \\
\cline { 2 - 3 } & $0.305 \mathrm{~mm}$ & 6.3 \\
\cline { 2 - 3 } & $0.303 \mathrm{~mm}$ & 6.3 \\
\hline \multirow{3}{*}{$\begin{array}{c}\text { Without considering spinning } \\
\text { craft experiment influenced by } \\
\text { tail jacking force }\end{array}$} & $0.310 \mathrm{~mm}$ & 6.3 \\
\cline { 2 - 3 } & $0.304 \mathrm{~mm}$ & 6.3 \\
\cline { 2 - 3 } & $0.305 \mathrm{~mm}$ & 6.3 \\
\cline { 2 - 3 } & $0.307 \mathrm{~mm}$ & 6.3 \\
\hline
\end{tabular}

\section{Conclusion}

The spinning craft experimental results tell that considering the deformation brought by tail jacking force has certain effect on dimension accuracy of the aluminum spinning products, but the impact is very tiny. We can completely ignore it if the requirement to dimension accuracy of spinning products is not high, on the side, through the contrast of the spinning surface products molding quality, find out it makes no difference if you consider the surface roughness of spinning products caused by the tail jacking force or not.

\section{References}

[1] Zhao Yunhao, Li Yanli. Technology and application of spinning. Beijing, 2008, Machinery Industry Press.

[2] Matsunok. Recent Research and Development in Metal Forming in Japan [J]. Mater, Process, Technol, 3(1997)51-54.

[3] Anon. Metal spinning in the auto motive industry [J]. Sheet Metal Industries, 18(1995)23-25.

[4] Xue Kemin, Wang Zhen, Lu Yan. Elasto-plastic FEM Analysis and Experimental Study of Diametral Growth in Tube Spinning [J], Mater, Process, Technol, 175(1997)69-72. 
[5] WANG Z T, XIE S S, JIN Q J. Elasto-plastic finite element analysis of hydrostatic extrusion with various mathematically contoured dies [J]. Proceedings of the 24th International Machine Tool Design and Research Conference, eds, Davies B [J]. 58(1983)51-58.

[6] HAYAMA, Hiroaki KUDO. Experimental Study of Tube Spinning [J]. Bulletion of JSME. 775(1979)167-170. 